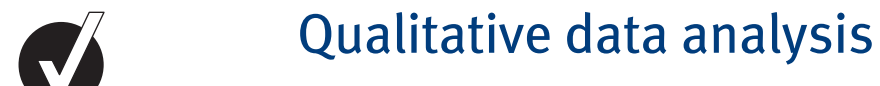

EDITOR'S

CHOICE

10.1136/ebnurs.2011.100352

Correspondence to:

Kate Seers

RCN Research Institute, School of Health \& Social Studies, University of Warwick, Coventry, CV4 7AL, Warwick, UK; kate.seers@warwick.ac.uk

\section{Kate Seers} text and can include images.
Good qualitative research uses a systematic and rigorous approach that aims to answer questions concerned with what something is like (such as a patient experience), what people think or feel about something that has happened, and it may address why something has happened as it has. Qualitative data often takes the form of words or

Qualitative research covers a very broad range of philosophical underpinnings and methodological approaches. Each has its own particular way of approaching all stages of the research process, including analysis, and has its own terms and techniques, but there are some common threads that run across most of these approaches. This Research Made Simple piece will focus on some of these common threads in the analysis of qualitative research.

So you have collected all your qualitative data - you may have a pile of interview transcripts, field-notes, documents and notes from observation. The process of analysis is described by Richards and Morse $^{1}$ as one of transformation and interpretation.

It is easy to be overwhelmed by the volume of data novice qualitative researchers are sometimes told not to worry and the themes will emerge from the data. This suggests some sort of epiphany, (which is how it happens sometimes!) but generally it comes from detailed work and reflection on the data and what it is telling you. There is sometimes a fine line between being immersed in the data and drowning in it!

A first step is to sort and organise the data, by coding it in some way. For example, you could read through a transcript, and identify that in one paragraph a patient is talking about two things; first is fear of surgery and second is fear of unrelieved pain. The codes for this paragraph could be 'fear of surgery' and 'fear of pain'. In other areas of the transcript fear may arise again, and perhaps these codes will be merged into a category titled 'fear'. Other concerns may emerge in this and other transcripts and perhaps best be represented by the theme 'lack of control'. Themes are thus more abstract concepts, reflecting your interpretation of patterns across your data. So from codes, categories can be formed, and from categories, more encompassing themes are developed to describe the data in a form which summarises it, yet retains the richness, depth and context of the original data. Using quotations to illustrate categories and themes helps keep the analysis firmly grounded in the data. You need to constantly ask yourself 'what is happening here?' as you code and move from codes, to categories and themes, making sure you have data to support your decisions.
Analysis inevitably involves subjective choices, and it is important to document what you have done and why, so a clear audit trail is provided. The coding example above describes codes inductively coming from the data. Some researchers may use a coding framework derived from, for example, the literature, their research questions or interview prompts, (Ritchie and Spencer ${ }^{2}$ ) or a combination of both approaches.

Qualitative data, such as transcripts from an interview, are often routed in the interaction between the participant and the researcher. Reflecting on how you, as a researcher, may have influenced both the data collected and the analysis is an important part of the analysis.

As well as keeping your brain very much in gear, you need to be really organised. You may use highlighting pens and paper to keep track of your analysis, or use qualitative software to manage your data (such as NVivio or Atlas Ti). These programmes help you organise your data - you still have to do all the hard work to analyse it! Whatever you choose, it is important that you can trace your data back from themes to categories to codes. There is nothing more frustrating than looking for that illustrative patient quote, and not being able to find it.

If your qualitative data are part of a mixed methods study, (has both quantitative and qualitative data) careful thought has to be given to how you will analyse and present findings. Refer to 0'Caithain et $a l^{3}$ for more details.

There are many books and papers on qualitative analysis, a very few of which are listed below. ${ }^{4-6}$ Working with someone with qualitative expertise is also invaluable, as you can read about it, but doing it really brings it alive.

Competing interests None.

\section{References}

1. Richards L, Morse J. Users Guide to Qualitative Methods Second edition. Thousand Oaks, CA: Sage 2007.

2. Ritchie J, Spencer E. Qualitative data analysis for applied policy research. In, Bryman A, Burgess RG, eds. Analyzing Qualitative Data. London: Routledge 1994.

3. O'Cathain, Murphy E Nicholl J. Three techniques for integrating data in mixed methods. BMJ 2010;341:c4587.

4. Bradley EH, Curry LA, Devers KJ. Qualitative data analysis for health services research: developing taxonomy, themes, and theory. Health Serv Res 2007;42:1758-72.

5. Gibbs GR. What and how to code, 2010. http://onlineqda.hud. ac.uk/Intro_QDA/how_what_to_code.php (accessed 21 October 2011).

6. Miles MB, Huberman AM. Qualitative Data Analysis. An Expanded Sourcebook. Second edition. Thousand Oaks, CA: Sage 1994. 